\section{SOLUÇÕES PARA - DESENVOLVIMENTO SUSTENTÁVEL dA AMAzôNIA}

\author{
Virgilio Viana \\ Emma Torres \\ Adalberto Val \\ Victor Salviati
}

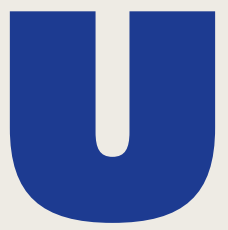

$\mathrm{m}$ dos principais desafios para a promoção do desenvolvimento sustentável é encontrar soluçôes práticas e economicamente viáveis. Isso se aplica tanto na escala global quanto regional. Na Amazônia este desafio é ainda mais sério. De um lado, existe um problema estrutural: o pequeno apoio dado às instituiçōes de ciência, tecnologia e inovação da região. De outro, destaca-se a complexidade de uma região que abriga uma enorme diversidade social, econômica, étnica, cultural, biológica e física.

Este artigo descreve uma recém lançada iniciativa, denominada "Rede de Soluções para o Desenvolvimento Sustentável da Amazônia" que faz parte da Rede de Soluçōes de Desenvolvimento Sustentável das Naçōes Unidas (na sigla em inglês, SDSN) (1). A SDSN foi lançada em 2012 para melhorar o conhecimento científico e tecnológico global para os desafios do desenvolvimento sustentável, incluindo a concepção e implementação da agenda global de desenvolvimento sustentável pós-2015. O conselho de liderança da rede SDSN apresentou o relatório ao secretário-geral da ONU, em junho de 2013, intitulado "Uma agenda de ação para o desenvolvimento sustentável" (2).

A Rede de Soluçôes para o Desenvolvimento Sustentável da Amazônia (SDSN-Amazônia) foi lançada em março de 2014, em Manaus. Desenvolver novas soluçōes e compartilhar soluções já existentes entre diferentes segmentos das sociedades amazônicas é algo que precisa ser estimulado. Esta é a missão da SDSN-Amazônia, da qual fazem parte instituições de pesquisa, empresas, organizações não governamentais e instituições governamentais dos oito países que compōem a Amazônia continental.

PREMISSAS E OBJETIVOS DA SDSN-AMAZÔNIA A concepção da SDSN-Amazônia parte de cinco premissas.

(i) Existem soluções já testadas e que ainda são pouco conhecidas na região. Portanto, dar mais visibilidade e disseminar estas soluçôes pode acelerar a construção de processos voltados para o desenvolvimento sustentável da região.

(ii) Existem recursos humanos altamente capacitados nas instituições da Amazônia. Portanto, apoiar estas instituições e suas parceiras no desenvolvimento de novas soluções é essencial para resolver os inúmeros gargalos que limitam o desenvolvimento sustentável da região.

(iii) Existe um rico saber etnoecológico junto às populações indígenas e povos tradicionais. Portanto, valorizar este saber, reduzir o processo de erosão cultural e criar pontes com o conhecimento científico e tecnológico é de importância estratégica.

(iv) A região abriga um setor privado empreendedor dinâmico, com forte capacidade de inovação tecnológica. Portanto, é necessário ampliar a capacidade de oferta de soluçōes desenvolvidas pelo setor empresarial, urbano e rural.

(v) É necessário incorporar as soluções existentes no processo de melhoria da eficiência das políticas públicas. Muitos erros de concepção e implementação de políticas públicas poderiam ser evitados com base no melhor aproveitamento das soluções já existentes.

A proposta da SDSN-Amazônia é quebrar a velha prática de importar e aplicar soluçóes de fora da Amazônia, sem as devidas adaptaçôes, para planejar o seu desenvolvimento. Historicamente isso tem resultado em fracassos ambientais, sociais e econômicos (3). A proposta do SDSN-Amazônia é fazer o oposto: mapear e disseminar soluções desenvolvidas pelas populaçōes amazônicas e suas instituiçôes de pesquisa, organizaçōes não governamentais, empresas e instituições governamentais. Esse desafio pode e deve contar com a colaboração de instituiçōes públicas e privadas de outras regiōes do planeta: não se trata de apoiar uma visão bairrista e provinciana ultrapassada. Porém, existe uma clara visão de que as instituições da Amazônia podem e devem desempenhar o papel de protagonistas desse processo.

O objetivo da SDSN-Amazônia é mobilizar centros de conhecimento da região para buscar soluções práticas e viáveis para os problemas do desenvolvimento sustentável da região amazônica. A participação nesta rede está aberta a universidades, centros de pesquisa, organizaçōes da sociedade civil, instituiçōes governamentais e a empresas dispostas a participar ativamente na concepção, pesquisa, desenvolvimento e implementação de soluções para o desenvolvimento sustentável na Amazônia.

CONTEXTO A bacia Amazônica comporta a maior floresta tropical do mundo (4). Sua área abrange cerca de $40 \%$ do continente sul-americano. A floresta amazônica abriga quase metade da biodiversidade do mundo (5-7). Milhōes de espécies, a maioria delas ainda não conhecidas pela ciência, habitam esta região (8). A bacia amazônica é também essencial para os ecossistemas por contribuir para a estabilidade do clima e produção de chuva nos níveis local, regional e global. Como exemplo, o rio Amazonas fornece cerca de 15\% de toda a água doce que entra nos oceanos em todo o mundo (9-11).

A bacia amazônica abrange oito países: Brasil, Bolívia, Peru, Equador, Colômbia, Venezuela, Guiana, Suriname e Guiana Francesa. Mais de 30 milhōes de pessoas, incluindo 350 grupos étnicos - dos quais, apenas no Brasil, cerca de 32 grupos ainda não foram contatados (12) - vivem na Amazônia e dependem dos recursos naturais para a produção de alimentos, vestuário e medicamentos tradicionais. A região é muito diversificada: indo de áreas agrícolas com alto grau de produtividade a áreas de extrema pobreza. A maioria da população vive em áreas urbanas da Amazônia (13; 14). A proporção da população urbana aumentou de $42 \%$, em 1970, para $71 \%$ da população total, em 2007 (15). Na Amazônia brasileira, de 1991 a 2010, a população urbana cresceu 29\% (16). 
A bacia amazônica está passando por grandes mudanças. As mudanças climáticas levam os ecossistemas florestais perto de seus respectivos pontos de inflexão (tipping point) (17-20). As principais causas do desmatamento incluem grandes projetos de infraestrutura (21), produção de carne bovina (22) e expansão agrícola de larga escala (23), a extração não sustentável dos recursos naturais (incluindo a mineração) (24), a exploração de petróleo e a extração ilegal de madeira (5). Há uma crescente consciência política e de compromisso dos governos e da sociedade civil da Amazônia para encontrar caminhos para o desenvolvimento sustentável da região (25). As principais realizaçōes incluem a redução significativa na taxa de desmatamento, especialmente no Brasil que diminuiu 84\% durante o período 2004-2012 (26). O estabelecimento de um vasto sistema de áreas protegidas, reservas indígenas, reservas extrativistas e outros tipos criaram um instrumento importante para reduzir a pressão sobre os recursos naturais $(23 ; 25$; $27 ; 28)$. Quase metade da região está classificada como área protegida natural ou territórios indígenas $(29 ; 30)$.

Vale notar que existe um histórico isolamento entre a Amazônia espanhola e portuguesa. Isso perdura desde o período colonial até os dias atuais. Existe também um isolamento geográfico entre os diversos contextos na Amazônia de cada país. Há pouco intercâmbio entre essas regiōes amazônicas. A falta de estradas e voos comerciais regulares, somadas à relativa baixa relação comercial e cultural são fatores importantes nesse isolamento. Novos eixos de integração, como a rodovia inter-oceânica Brasil-Peru, começam a mudar esse quadro. Por outro lado, o aumento da integração traz outros desafios, como a criação e a potencialização de vetores de pressão sobre os recursos naturais como as atividades ilegais de desmatamento e garimpo $(5 ; 24)$.

A Amazônia é uma região de grande importância para o equilíbrio global, especialmente na atual era que vivemos - o Antropoceno. Neste contexto, marcado pelas mudanças climáticas e pela aproximação dos limites planetários (31), é cada vez mais importante o papel das florestas e demais ecossistemas da Amazônia para o apoio à resiliência global.

ATIVIDADES A rede SDSN-Amazônia irá desenvolver os seguintes tipos de atividades:

I) Identificar e promover iniciativas de soluções para o desenvolvimento sustentável na região amazônica.

A rede irá identificar e promover iniciativas concretas de soluções que envolvem novas tecnologias, modelos de negócio, mecanismos institucionais inovadores e políticas públicas promissoras. Essas iniciativas deverão ter um impacto transformador sobre o desenvolvimento sustentável na região.

II) Construir uma plataforma digital e online para compartilhar conhecimento da e para a Amazônia.

O objetivo é ter uma plataforma de acesso aberto, utilizando uma ferramenta de georeferenciamento que permita a troca de informaçōes sobre as soluçōes sustentáveis. A seleção dessas soluçōes será feita de acordo com um conjunto de critérios dentro das diretrizes da SDSN. Esta iniciativa criará uma plataforma online de livre acesso para informaçôes relativas ao desenvolvimento sustentável na Amazônia e demais regiōes.

Muitas soluçôes para o desenvolvimento sustentável estão sendo concebidas e implementados com sucesso na Amazônia. No entanto, atualmente, há poucas ou nenhuma plataforma para aglutinar e disponibilizar de maneira organizada essas soluçōes. A plataforma aqui proposta terá um efeito multiplicador na difusão de soluçôes para a Amazônia e o mundo. Esta atividade tem como base a experiência desenvolvida pela Fundação Amazonas Sustentável, em colaboração com o Google Outreach e a Ritual Cultura, Meio Ambiente e Entretenimento, para exibir as mais diversas informações de atividades de desenvolvimento sustentável implementadas em comunidades ribeirinhas no estado do Amazonas.

A plataforma irá reunir as melhores informações e conhecimentos gerados por instituiçôes científicas, de pesquisa acadêmica, governos nacionais e locais, empresas e instituições do terceiro setor nos níveis local, nacional e regional. A rede SDSN-Amazônia irá identificar essas soluçōes por meio de convites públicos e prêmios. O processo de seleção será baseado em metodologia clara e transparente, desenvolvida em parceria com instituiçōes e membros da rede. Quando pronto, esse processo será amplamente divulgado pela SDSN-Amazônia e seus parceiros. Adicionalmente, a rede também participará de reuniōes e eventos relacionados aos temas tratados. Isso inclui eventos como reuniōes da Associação Internacional de Universidades, do Fórum Mundial da Ciência, e de Conferências do Clima, entre outros.

III) Apoiar, tecnicamente com base em evidências, os tomadores de decisōes na implementação de políticas públicas e soluções em prol do desenvolvimento sustentável.

A redeSDSN-Amazônia pretende engajar tomadores de decisōes com o objetivo de abordar as questôes técnicas e prioridades identifi- 
cadas por seus parceiros e apoiar a proposição de arranjos, atividades e soluções no nível político. Isto caberá tanto para multiplicar ações já existentes (para potencializar impactos positivos e gerar escala) quanto para elaborar inovações. Adicionalmente, a SDSN-Amazônica visa ser uma ponte entre os governos e a sociedade civil e o setor privado - dinamizando o fluxo de informações - para identificar e desenvolver estratégias para apoiar a implementação de políticas em cooperação para o desenvolvimento sustentável na região.

Por fim, a rede também visa promover a cooperação Sul-Sul. Através da troca de experiências e liçôes aprendidas da Amazônia, há a oportunidade promissora de interação com outras regiōes de florestas tropicais, como a bacia do Congo.

GOVERNANÇA A rede SDSN-Amazônia faz parte da iniciativa internacional SDSN liderada pelo professor Jeffrey Sachs. A Fundação Amazonas Sustentável (FAS) atuará como secretaria executiva do SDSN-Amazônia.

Uma vez que outras instituições-membro se engajem, estas assumirão tarefas específicas de coordenação na rede. A governança dessa rede deve ser representativa e flexível. No primeiro momento há um esforço da rede em mobilizar instituições de todos os países da bacia amazônica e garantir a sua plena participação.

A rede SDSN-Amazônia será liderada por três co-presidentes e por quatro comitês: científico, político-estratégico, empresarial e da sociedade civil organizada.

O comitê científico será composto por pesquisadores de renome da região, das mais diversas instituiçôes. Os termos de referência desse comitê ainda serão definidos pela rede e deverão: fornecer subsídios técnicos e científicos, identificando os desafios e as intervençôes prioritárias para a região Amazônica; identificar, avaliar e apoiar o desenvolvimento de soluções para o desenvolvimento sustentável na Amazônia de maneira multidisciplinar; mobilizar e convocar populaçōes tradicionais, indígenas, entre outras, para interagir nos processos da rede, e partilhar ações e conhecimentos.

O comitê político-estratégico será composto por tomadores de decisões e gestores de agências internacionais. Os termos de referência desse comitê ainda serão definidos pela rede e deverão: apoiar a definição e a execução de estratégias e ações prioritárias da rede; identificar, avaliar e apoiar o desenvolvimento de soluçôes para o desenvolvimento sustentável na Amazônia de maneira multidisciplinar; mobilizar e convocar populações tradicionais, indígenas, entre outras, para interagir nos processos da rede, e partilhar açôes e conhecimentos.

O comitê empresarial será composto por gestores de grandes empresas com atuação na região amazônica. Os termos de referência desse comitê ainda serão definidos pela rede e deverão: apoiar a execução de estratégias e açōes prioritárias da rede; identificar, avaliar e apoiar o desenvolvimento de soluçôes para o desenvolvimento sustentável na Amazônia de maneira multidis- ciplinar; mobilizar e convocar populações tradicionais, indígenas, entre outras, para interagir nos processos da rede, e partilhar açôes e conhecimentos.

Já o comitê do terceiro setor será composto por gestores e representantes das organizações da sociedade civil. Os termos de referência desse comitê ainda serão definidos pela rede e deverão: apoiar a definição de estratégia e ações prioritárias da rede; identificar, avaliar e apoiar o desenvolvimento de soluçôes para o desenvolvimento sustentável na Amazônia de maneira multidisciplinar; mobilizar e convocar movimentos sociais, populaçōes tradicionais, indígenas, entre outras, para interagir nos processos da rede, e partilhar ações e conhecimentos.

\section{RESULTADOS DO EVENTO DE LANÇAMENTO DO SDSN-AMAZÔNIA}

O lançamento da rede SDSN-Amazônia aconteceu em Manaus, Brasil, em 18 de março de 2014, e contou com representantes dos países da bacia amazônica dos mais diversos setores (32). Os encaminhamentos e os próximos passos definidos no evento são:

- Materializar o programa de trabalho da rede SDSN-Amazônia com sua estratégia, seus objetivos (geral e específicos), cronograma e orçamento;

- Consolidar a rede pela formalização de membros, dos comitês, da secretaria executiva e a governança da rede;

- Identificar e promover soluções sustentáveis pela elaboração de seus critérios, criação dos "prêmios para soluçôes sustentáveis”, e lançar a plataforma online para sua respectiva divulgação;

- Desenvolver cursos de curta e longa duração em desenvolvimento sustentável para universidades e centros de pesquisa da Amazônia;

- Criar grupos de pesquisadores qualificados para oferecer cursos periódicos e colaborarem na execução de projetos de pesquisa em sustentabilidade na Amazônia;

- Promover a troca de experiências e informaçóes por meio de seminários, debates qualificados e eventos técnicos.

CONCLUSÕES Existem diversas soluções setoriais para a promoção do desenvolvimento sustentável, mas algumas destas não são suficientemente conhecidas, divulgadas e desenvolvidas. Este é um problema global, não apenas da Amazônia. Há, por meio da rede SDSN-Amazônia, a oportunidade de utilizar a tecnologia da informação para conectar os desenvolvedores de soluções com aqueles que demandam este conhecimento.

Existem soluções para o manejo sustentável do pirarucu, a criação de tambaqui em cativeiro, a melhoria da educação, o atendimento à primeira infância, a geração de energia renovável e de qualidade para comunidades isoladas, a agricultura com sistemas agroflorestais, boas práticas para a produção mineral etc. Fazer com que essas soluções sejam conhecidas, e que haja a interação entre atores, são alguns dos grandes desafios para promover um estilo de desenvolvimento capaz de melhorar a qualidade de vida das pessoas e ao mesmo tempo promover a conservação da floresta da Amazônia. 
É necessário mobilizar todos os segmentos das sociedades amazônicas para acelerar a disseminação de soluçôes existentes e o desenvolvimento de outras novas. E, ainda, é necessário ampliar as pontes e fomentar a cooperação entre diferentes segmentos das sociedades para a construção de caminhos legítimos para o desenvolvimento sustentável da Amazônia.

A SDSN-Amazônia pretende valorizar o conhecimento das populaçōes e instituiçōes amazônicas. Esta valorização vem por meio do incentivo aos mais diversos tipos de parcerias e arranjos institucionais para promover e potencializar soluçôes para o desenvolvimento sustentável. Esta rica rede de conhecimentos e instituições terá impactos positivos não só na Amazônia mas também em outras regiôes do mundo - atestando a importância global de se pregar o desenvolvimento sustentável na e para a Amazônia.

Virgilio Viana é superintendente geral da Fundação Amazonas Sustentável.

Emma Torres é assessora especial para biodiversidade e serviços ecossistêmicos do Programa das Naçōes Unidas para o Desenvolvimento (Pnud) e co-presidente do SDSN-Amazônia.

Adalberto Val é pesquisador do Instituto Nacional de Pesquisas da Amazônia (Inpa) e co-presidente do SDSN-Amazônia.

Victor Salviati é coordenador de projetos especiais da Fundação Amazonas Sustentável e secretário executivo interino da SDSN-Amazônia.

\section{NOTAS E REFERÊNCIAS BIBLIOGRÁFICAS}

1. http://unsdsn.org/.

2. SDSN. "Uma agenda de ação para o desenvolvimento sustentável" In: Sustainable Development Solutions Network, 2013. Disponível em http://goo.gl/uBiq7g.

3. Viana, V. "A Amazônia e o interesse nacional" In: Revista Política Externa, volume, (19), 2011.

4. FAO. "Global forest resources assessment". 2010. Disponível em http://www.fao.org/docrep/013/i1757e/i1757e.pdf

5. Malhi, Y.; Roberts, J. T.; Betts, R. A.; Killeen, T. J.; Li, W.; Nobre, C. A. “Climate change, deforestation, and the fate of the Amazon". In: Science, 319(5860), p 169-172, 2008.

6. Betts, R. A.; Malhi, Y.; Roberts, J. T. "The future of the Amazon: new perspectives from climate, ecosystem and social sciences". In: Philosophical Transactions of the Royal Society B: Biological Sciences, 363(1498), p 1729-1735, 2008. Disponível em http://rstb.royalsocietypublishing.org/content/363/1498/1729.short

7. Myers, N.; Mittermeier, R. A.; Mittermeier, C. G.; da Fonseca, G. A.; Kent, J. "Biodiversity hotspots for conservation priorities". In: Nature, 403(6772), p 853-858, 2000. Disponível em http://www.nature. com/nature/journal/v403/n6772/full/403853a0.html

8. Hopkins, M. J. "Modelling the known and unknown plant biodiversity of the Amazon Basin" In: Journal of Biogeography, 34(8), 1400-1411, 2007. Disponível em http://onlinelibrary.wiley.com/doi/10.1111/j. 1365-2699.2007.01737.x/abstract

9. Wohl, E. E. "Hydrology and discharge". In:Large rivers: geomorphology and management. Wiley, p 29-44, 2007.

10. Dai, A.; Trenberth, K. E. "Estimates of freshwater discharge from continents: latitudinal and seasonal variations". In: Journal of Hydrometerology. American Meteorological Society, p 660-687. 2002.
11. Dai, A.; Trenberth, K. E. "New estimates of continental discharge and oceanic freshwater transport". In: AMS Symposium on Observing and Understanding the Variability of Water in Weather and Climate. 2003.

12. De acordo com a Fundação Nacional do Índio (Funai) em http://www. funai.gov.br/indios/fr_conteudo.htm.

13. IBGE. "Sinopse do Censo Demográfico - 2010". In: Instituto Brasileiro de Pesquisa e Estatística. 2010. Disponível em http://www.ibge.gov. br/home/estatistica/populacao/censo2010/sinopse.pdf

14. Barthem, R. B.; Charvet-Almeida, P.; Montag, L. F. A.; Lanna, A. E. "Global International waters assessment Amazon basin, GIWA regional assessment 40 b". In: GIWA Regional assessment reports: United Nations Environment Programme, p 01-76, 2004.

15. Castelani, S. A.; Igliori, D. "Urbanization and growth in the Brazilian Amazon". In: Núcleo de Economia Socioambiental/USP: working paper, p1-26, 2012.

16. IBGE, 2011.

17. Leadley, P.; Pereira, H. M.; Alkemade, R.; Fernandez-Manjarrés, J. F.; Proença, V.; Scharlemann, J. P. W.; Walpole, M. J. “Biodiversity scenarios: projections of 21 st century change in biodiversity and associated ecosystem services". In: Secretariat of the Convention on Biological: Technical Series no. 50, p 1-132, 2010. Disponível em http://www.miljodirektoratet.no/old/dirnat/multimedia/45974/CBD-TS50-GBO3-Scenarios-Digital.pdf.

18. World Bank. "Climate change and clean energy initiative". In: Assessment of the Risk of Amazon Dieback, 2010. Disponível em http://www. bicusa.org/en/Document.101982.aspx

19. Nobre, C. A., and L. D. Borma. "'Tipping points' for the Amazon forest". Current Opinion in Environmental Sustainability 1:28-36. 2009.

20. Nepstad, D. C.; Stickler, C. M.; Soares-Filho, B.; Merry, F. “Interactions among Amazon land use, forests and climate: prospects for a near-term forest tipping point". In: Philosophical Transactions of the Royal Society B: Biological Sciences, 363(1498), 1737-1746, 2008.

21. Perz, S. G.; Qiu, Y.; Xia, Y.; Southworth, J.; Sun, J.; Marsik, M.; Baraloto, C. "Trans-boundary infrastructure and land cover change: Highway paving and community-level deforestation in a tri-national frontier in the Amazon". In:LandUse Policy, 34,27-41.2013. Disponível em http:// www.sciencedirect.com/science/article/pii/S026483771300029X

22. Mann, M. L.; Kaufmann, R. K.; Bauer, D. M.; Gopal, S.; Nomack, M.; Womack, J. Y.; Soares-Filho, B. S. "Pasture conversion and competitive cattle rents in the Amazon". In: Ecological Economics, 97, p 182-190. 2014. Disponível em http://www.sciencedirect.com/science/article/ pii/S0921800913003558

23. Amin, A.; Choumert, J.; Combes Motel, P.; Combes, J.L.; Kere, E.N.; Ongono-Olinga, J.G.; Schwartz, S. "A spatial econometric approach to spillover effects between protected areas and deforestation in the Brazilian Amazon". In: Etudes et Documents, 6, p 2-27. 2014. Disponível em http://halshs.archives-ouvertes.fr/docs/00/96/04/76/ PDF/2014.06.pdf

24. Asner, G. P.; Llactayo, W.; Tupayachi, R.; Luna, E. R. "Elevated rates of gold mining in the Amazon revealed through high-resolution monitoring". In: Proceedings of the National Academy of Sciences, 110(46), 18454-18459, 2013. Disponível em http://www.pnas.org/content/110/46/18454.full.pdf+html 
25. Nolte, C.; Agrawal, A.; Silvius, K. M.; Soares-Filho, B. S. “Governance regime and location influence avoided deforestation success of protected areas in the Brazilian Amazon". In: Proceedings of the National Academy of Sciences, 110(13), 4956-4961. 2013. Disponível em http:// www.pnas.org/content/110/13/4956.short

26. Inpe. Taxas anuais de desmatamento - 1998 até 2013. 2014. Disponível em http://www.obt.inpe.br/prodes/prodes_1988_2013.htm. Acessado em 8 de junho de 2014.

27. Ferreira, L. V.; Venticinque, E.; Almeida, S. "O desmatamento na Amazônia e a importância das áreas protegidas". In: Estudos Avançados, 19(53), 157-166, 2005. Disponível em http://www.scielo.br/scielo. php?pid=S0103-40142005000100010\&script=sci_arttext\&tIng=pt.

28. Naughton-Treves, L.; Holland, M. B.; Brandon, K. "The role of protected areas in conserving biodiversity and sustaining local livelihoods". In: Annu. Rev. Environ. Resour., 30, 219-252, 2005. Disponível em http://www.annualreviews.org/doi/abs/10.1146/annurev.energy.30.050504.164507

29. Soares-Filho, B.; Moutinho, P.; Nepstad, D.; Anderson, A.; Rodrigues, H.; Garcia, R.; Maretti, C. "Role of Brazilian Amazon protected areas in climate change mitigation". In: Proceedings of the National Academy of Sciences, 107(24), 10821-1082, 2010. Disponível em http://www. pnas.org/content/107/24/10821.full.pdf+html

30. Chape S.; Spalding M.; Jenkins M. D. "The World's Protected Areas". In:UNEP World Conservation Monitoring Centre: University of California Press, 2008. Disponível em https://ia600503.us.archive.org/7/ items/worldsprotecteda08chap/worldsprotecteda08chap.pdf

31. Rockström et al. "A safe operating space for humanity". In: Nature, 461, 472-475, 2009. Disponível em http://www.nature.com/nature/ journal/v461/n7263/full/461472a.html

32. Veja informações no site: http://sdsn-amazonia.org/

\section{COOPERACT̃O EM GIÊNCIA, TEGNOLOGAA E NOVACÄO E EDUGACAO NOS PAÍÉES AMAZONICOS}

\author{
Claudio Ruy Vasconcelos da Fonseca
}

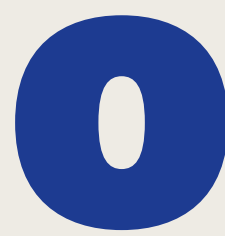

Fórum Mundial de Ciência ocorrido no Rio de Janeiro em novembro de 2013 discutiu a necessidade de mudanças nas relações internacionais, considerando que os sistemas econômico e de transporte quase tornaram extintas as distâncias horizontais que separavam os mercados planetários. Tal situação provocou mudanças profundas nas dinâmicas ambientais e no comportamento social, transformando problemas, outrora localizados geograficamente, em grandes enigmas globais. A complexidade dos problemas ambientais está a requerer soluçōes inovadoras que, por sua vez, exigem articulações multinacionais (pesquisas e tecnologias) e mudanças no domínio diplomático-científico.

A produção do conhecimento vem sendo considerada tarefa central para quase todos os países e, neste contexto, a transformação através de uma sociedade do conhecimento é o caminho para assegurar prestígio e competitividade (1). Pesquisa passa a ser encarada como parte da atividade econômica requerendo ações de agregação nos vários níveis espaciais.

No caso específico dos países amazônicos, estudos que avaliam pressupostos desta natureza são escassos e pouco é discutido sobre a dimensão regional da produção colaborativa de conhecimento, a despeito de se considerar a cooperação relevante às políticas de ciência e tecnologia dos países. Indicadores de produção de ciência no século XXI demonstram que colaboração científica é a característica predominante no mundo (2). Trabalhos em coautoria formam $50 \%$ de toda publicação científica atual (3).

O PROCEDIMENTO DO BRASIL A literatura que analisa a cooperação científica internacional, do ponto de vista político e estratégico, enxerga alguns impulsionadores para as publicaçōes em coautoria entre países nos vários continentes (3): i) A emergência de alguns países (ex. Brics) com desenvolvimento de pesquisas e tecnologias reconhecidas como compatíveis com os padrões internacionais; ii) o crescimento do debate sobre as mudanças globais, sustentabilidade energética e segurança em saúde; iii) a globalização da pesquisa e desenvolvimento acelerada pela mobilidade dos pesquisadores, atualmente mais visível na pesquisa industrial; iv) o crescimento demográfico e o decréscimo de capital humano para ciência e engenharia, resultando na escassez de talentos, o que torna a colaboração uma via para atrair talentos de países parceiros; v) a ampliação dos debates sobre políticas e aspirações para prover mais massa crítica e perfil internacional de excelência em pesquisa e, nesta conjuntura, a parceria com o melhor desempenha papel decisivo. 